\title{
Finite element analysis model of anti-vibration performance of steel structure buildings
}

\author{
FanSu \\ (Sichuan college of architecture technology Structure technology center Sichuan Deyang \\ 618000) \\ fansuhy@163.com
}

Keywords: steel structure; anti-vibration; finite element analysis model

\begin{abstract}
. in order to study the anti-vibration performance of the steel structure buildings, the finite element analysis model was established. On the basis of reasonable selection of the type of finite element, the failure modes, ultimate load, maximum plastic rotation, ductility and energy dissipation performance of the type of flange weakening node of steel structure buildings are obtained, to get the load - displacement hysteresis back curve and local failure modes of the steel structure buildings. the anti-vibration performance finite element analysis model of steel structure buildings is established and to make compared analysis with the anti-vibration performance of standard steel structure buildings in bearing capacity, stiffness, hysteretic behavior, degradation characteristics and failure modes and analyzed. The analysis results show that the model has a certain role in promoting the improvement of seismic design code of steel structure and engineering application of high strength steel.
\end{abstract}

\section{Introduction}

Steel structure buildings have the characteristics of fast construction speed, high degree of industrialization, excellent technical and economic indexes, and superior seismic performance compared to other construction materials, so it can widely be used in various construction fields [1-3]. Due to the different forms of steel structures, there are obvious difference of their seismic performances. Therefore, to analyze and select steel structure reasonably, enhance the ductility of steel structure, and ensure that the steel structure in different magnitude might achieve the seismic fortification goal, become the main problems which is urgent to solve in the architecting research field [4-6]. To establish the finite element analysis model for anti-vibration performance of steel structure buildings, can simulate and analyze the load-displacement hysteresis back curve and local failure modes of different steel structure buildings, which is an effective way to solve the above mentioned problem, causeing the attention of many experts and scholars [7-9]. Because to establish the finite element analysis model for anti-vibration performance of the steel structure buildings has profound significance for the development, it has become a focus of research in this field, and has received extensive attentions. There has also been a lot of good methods [10].

In recent years, in foreign countries, research on the anti-vibration performance of steel structure buildings is more, and in the project, it has been applied extensively, but study on the anti-vibration performance of steel structure buildings in domestic is less. The collected information is insufficient, although the design concept of bone shaped connecting is proposed in current seismic code, how to implement specifically, are not yet make specific provisions and instructions. In this paper, on the basis of reasonable selection of the type of finite element, the failure modes, ultimate load, maximum plastic rotation, ductility and energy dissipation performance of the type of flange weakening node of steel structure buildings are obtained, to get the load - displacement hysteresis back curve and local failure modes of the steel structure buildings. the anti-vibration performance finite element analysis model of steel structure buildings is established and to make compared analysis with the anti-vibration performance of standard steel structure buildings in bearing capacity, stiffness, hysteretic behavior, degradation characteristics and failure modes and analyzed. The 
analysis results show that the model has a certain role in promoting the improvement of seismic design code of steel structure and engineering application of high strength steel.

\section{Finite element model}

\subsection{Test model}

In the formation of the finite element analysis model for anti-vibration performance of steel structure buildings, different RBS joint connection specimens are made respectively according to the different flange weakening parameters of steel structure building, as shown in figure 1 . The distance $\alpha$ between the starting point in the weakened zone of specimens to the cylinder are taken as $110 \mathrm{~mm}(0.73 b f)$ and $70 \mathrm{~mm}(0.47 b f)$ respectively. The length $b$ of weakening zone are taken as $180 \mathrm{~mm}\left(0.60 h_{b}\right)$ and $240 \mathrm{~mm}\left(0.80 h_{b}\right)$, and the maximum cutting depth $c$ of a side of flange are $35 \mathrm{~mm}(0.23 b f)$ and $40 \mathrm{~mm}(0.23 b f)$ respectively. Among them, $b_{f}$ and $h_{b}$ are the height of the flange width and the beam section of construction beam respectively. The weaken parameters are shown in figure 1. In order to connect with the traditional joint, a common joint specimen is also produced. the joint domain of all joints are made up of steel plate to make thickness, showing in figure 1. The geometric dimension of the specimen is shown in Figure 2, where $p$ represents the external load of the end of beam, and the meaning of $a$ and $b$ is the same as that of $a$ and $b$ in Figure 1. In the test, in all beam column of specimens, Q235 hot rolled H-section steel is selected, the column section is HW200 $\times 200$, the thickness of web and flange are respectively for $8 \mathrm{~mm}$ and $12 \mathrm{~mm}$, the beam section is HN300 $\times 150$, and the thickness of web and flange are respectively for $6.5 \mathrm{~mm}$ and $9 \mathrm{~mm}$.

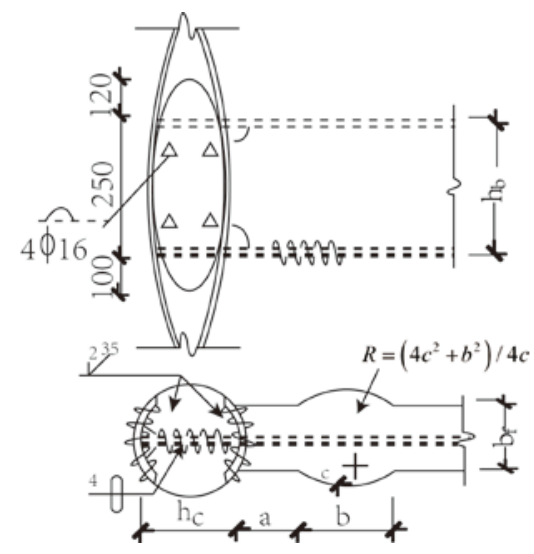

Figure 1 the detail structure of the flange weaken joint connection of the steel structure buildings

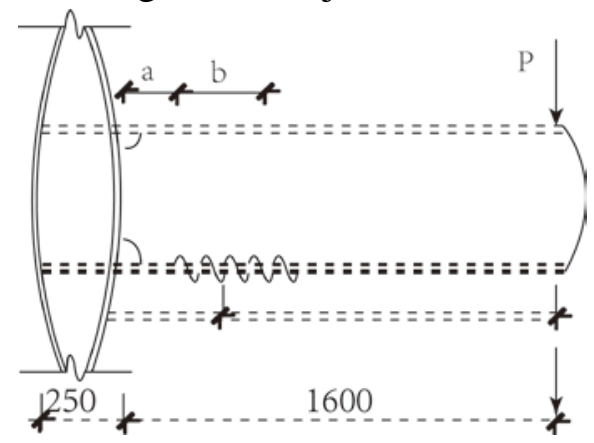

Figure 2 Schematic diagram of the geometry of specimen

The general finite element program ANSYS is used to establish the 3D model for the the flange weaken joint specimens of the steel structure building and the whole welded joint specimens in the test, and then the nonlinear finite element analysis is carried out.

\subsection{Selection of material parameters of specimens}

Specimens use Q235 steel, the trilinear model by considering the strengthening phase and descending phase is used for the description of stress-strain relationship, it can better meet the true 
stress - strain relationship of steel showed in one-way tensile test, and correctly describe the strength degradation phenomenon of strength hardening and exceed the limit strain after steel yielding. The detail is as shown in Figure 3.

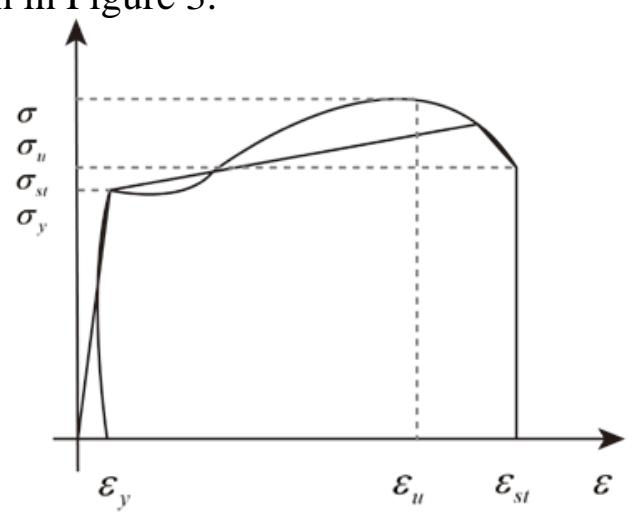

Figure 3 trilinear model of material stress-strain

\subsection{Unit selection and mesh generation}

In the finite element model division, the 45 solid elements with eight joints is selected for column to make mapping mesh generation. The 20 joints solid 95 solid elements with high accuracy are selected for the beam in the reduced area of beam flange to make free mesh generation. For the other parts of beam, the eight joints solid 45 solid elements are mapped mesh generation, and for the welding, 10 joints 92 solid elements are made free mesh generation. The finite element model is shown in figure 4.

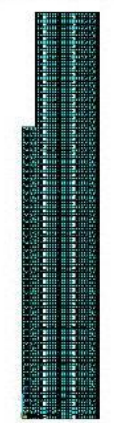

Figure 4 finite element model of steel structure buildings

\section{4 boundary conditions and loading}

When in the earthquake action, the anti-bending position of the beam column in steel frame is approximately in the middle of the component, and the finite element analysis is the same as the test, half span is to take for the beam, a layer of half is take for each up and down column. In this paper, in the whole process of the ANSYS simulation analysis, the displacement of the load for the beam is applied. Initial displacement is $20 \%$ of the yield displacement, each displacement incremental is about 20\% every time. Before yield, each load is made cycle 1 time; after specimens yielding, the 2 times, 3 times and 4 times yield displacement are successively applied, and each load makes 2 times cycles, the loading system is as shown in Figure $4, \Delta$ y is yield displacement.

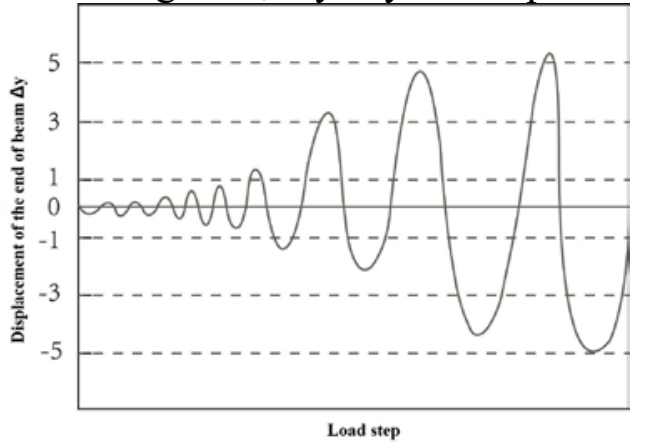

Figure 5 loading system 
In finite element analysis, under reciprocating load, specimen ST-1 to ST-6 have experienced the elastic stress stage, the elastic plastic stress stage, and the plastic hinge formation and the development stage, to reach the limit load of specimen and be failure, in good agreement with the experimental phenomena. The most obvious difference between RBS joint specimen ST- 1 to ST- 5 and ordinary joint specimen ST-6 is in the plastic hinge forming region. The plastic hinge of RBS joint specimen is on flange weakening region, and that of ordinary joint specimen in a beam butt weld region.

\section{Load-bearing Performance analysis of joints with different forms of steel structure}

The initial bending defects of specimen is obtained through the extraction of lowest order overall instability mode of components and correction of the joint coordinates of the model. According to the rule of the specification of steel structure, the maximum value of model's initial deflection is $1 / 1000$ beam length. In the initial deformation amplitude value $w_{B}$ and $w_{F}$ of plate strictly follows the relevant provisions for the allowable deviation of steel members assembled in the standard "Code of construction quality acceptance of steelwork engineering" (GB50205-2001). and then to make comparative analysis with the anti-vibration performance of standard steel structure buildings in bearing capacity, stiffness, hysteretic behavior, degradation characteristics and failure modes:

(1) Comparative analysis of load bearing performance

The structural form of the joint has little effect on the stiffness and bearing capacity, which is consistent with the original intention of changing structure.

(2) Comparative analysis of hysteretic performance

Apart from the long groove joint, other joint s' hysteretic curves are full.

(3) Comparative analysis of degradation characteristics

In stage 1 , it has no obvious degradation phenomenon, hysteresis loop basically coincides with the monotone curve; in stage 2, due to plastic strain accumulation, it leads to local buckling and rapid degenerative changes; in stage 3 , the degradation is again into the gentle, and with the increase of the displacement, it appears gradually calm and steady degradation and the curve finally tends to be consistent, and finally, the degradation is stable.

(4) Comparative analysis of failure modes

The local structure changes the position of the plastic hinge, the position and the length of the plastic hinge of the standard type joint can also make the base of beam to be included in the plastic hinge. The stress of the base is very large, and the brittle failure is easy to occur.

\section{Conclusions}

The nonlinear finite element analysis model for welding joint in steel frame is established, and to make compared analysis with the anti-vibration performance of standard steel structure buildings in bearing capacity, stiffness, hysteretic behavior, degradation characteristics and failure modes and analyzed, to get that the nonlinear finite element calculation model of joints in steel frame has good agreement with the typical experiment results in macroscopic failure pattern and local buckling and verify the reasonableness of applied model adopted in the element type, material constitutive and initial imperfections. Considering the seismic effect, building requirements and economic indicators, the weakening of the node is not too much to weaken the bearing capacity and stiffness of the node, and the hysteretic performance is stable, so the energy consumption of all nodes is relatively close.

\section{References}

[1] Chen Jie, Zheng Tingyin, Wu Xinghua. Finite element analysis for seismic performance of L shaped column of steel reinforced concrete [J]. Steel structure, 2014, 29 (3): 18-22.

[2] Chen Shaojie, Han Hangbo. The seismic performance of steel structure residential housing 
system [J]. Journal of Xi'an University of Science and Technology, 2014, 34 (1): 43-47.

[3] Li Feng, Li Fashan. Dynamic characteristics and seismic analysis of Kiewitt spherical reticulated mega structure [J]. Steel structure, 2015, 30 (3): 7-11.

[4] Zheng Shansuo, Xu Qiang, Tian Jin, et al. Seismic behavior analysis of welded joints by considering the influence of local structure form [J]. Engineering mechanics, 2014 (10): 136-142.

[5] Zhou Qian, Yan Weiming, Ji Jinbao. Seismic performance Analysis of steel structure for the Ling Zhao Xuan in Imperial Palace [J]. Journal of earthquake engineering, 2015 (1): 106-113.

[6] Zhu Yu, Li Qicai, Ji Rui. Research on seismic behavior of self-reset structure with steel plate shear wall energy consumption [J]. Development of Building steel structure, 2015, 17 (4): 19-26.

[7] Zhang Kun, Zhang Hongya. The influence of the setting position of the steel reinforced concrete beam transfer story on the structural seismic performance [J]. Steel structure, 2014, 29 (8): 32-35.

[8] Lu Senqiang, Fang Youzhen, Yao Jiangfeng, et al. Finite element analysis of seismic performance of interior joint of innovative PEC column-steel beam with welded split-tee connection [J]. Journal of Lanzhou University of Technology, 2015, 41 (1): 128-134.

[9] Dai Yujuan, Cheng Xiang, Yang Xianhai, et al. Micro Cutting Performance Simulation Analysis Based on Finite Element Method [J]. Computer simulation, 2014, 31 (3): 243-247.

[10] Wu Xiaodong, Sheng Meiping, Zhang Anfu, et al. An energy equivalent method for Young's modulus based on finite element analysis [J]. Electronic design engineering, 2014, 22 (13): 41-43. 